\title{
Natural Polymer Based Mucoadhesive Hydrogel Beads of Nizatidine: Preparation, Characterization and Evaluation
}

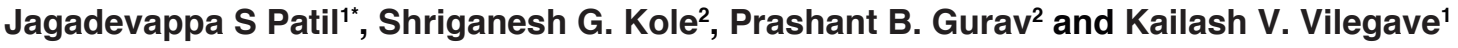 \\ ${ }^{1}$ Department of Pharmaceutics, VT's S. S. Jondhle College of Pharmacy, Asangoan, Tal. Shahapur, Dist. Thane-421 601, Maharashtra, \\ India. \\ 2Department of Pharmaceutics, SVERI's College of Pharmacy, Gopalpur-Ranjani Road, Gopalpur, Pandharpur-413 304, Maharashtra, \\ India.
}

\begin{abstract}
Background: Dosage forms which precisely control the release rates and targets drugs to a specific body site have made enormous impact in the formulation and development of novel drug delivery systems. Methods: A prolonged release mucoadhesive hydrogel system of nizatidine was prepared by ionotropic gelation and polyelectrolyte complexation technique using natural, biodegradable polymers with or without chitosan. Prepared formulations were subjected to in vitro evaluation and several characterization studies. Results: Formulations with chitosan showed good drug content, swelling index and mucoadhesive strength when compared to batches containing alginate alone. The drug in formulations found to be intact and compatible with polymers used and surface morphology of prepared beads were found satisfactory. Two optimized formulations containing alginatechitosan shows Higuchi model and perfect zero order release. All the formulations with copolymer showed better sustained the drug release when compared with formulations without chitosan. Conclusion: Alginate-chitosan beads prepared by ionotropic geltion and polyelectrolyte complexation method found to be better than ionically cross linked alginate beads alone. Therefore, dual cross-linked beads are promising carrier for oral control release.
\end{abstract}

Key words: Chitosan, ionotropic gelation, Mucoadhesion, Nizatidine, Sodium alginate.

\section{INTRODUCTION}

Multiparticulate systems have been paid considerable attention since several years in controlling and sustaining of release rate of many active pharmaceutical ingredients. And use of natural biodegradable polymers as rate controlling agents also has been enormously increased. Recently, dosage forms that can precisely control the release rates and targets drugs to a specific body site have made enormous impact in the formulation and development of novel drug delivery systems. Oral multiunit dosage forms such as microcapsules and microspheres have received much attention as modified/ controlled drug delivery systems for the treatment of various diseases without major side effects. Additionally, the beads maintain functionality under physiological condi- tions, can incorporate drug to deliver locally at high concentration ensuring that therapeutic levels are reached at the target site while reducing the side effects by keeping systemic concentration low. It will therefore be advantageous to have means for providing an intimate contact of the drug delivery system with microbeads. ${ }^{1}$

The suppression of gastric acid secretion with anti-secretary agents has been the mainstay of medical treatment for patients with acid-related disorders. The suppression of gastric acid secretion achieved with $\mathrm{H}_{2}$ receptor antagonists has, however, proved to be suboptimal for effectively controlling acidrelated disorders, especially for healing erosive oesophagitis and for the relief of reflux
Submission Date : 03-12-2014 Revision Date : :22-06-2015 Accepted Date : :19-07-2015

DOI: $\mathbf{X X X}$

Correspondence Address Mr. Jagadevappa S. Patil Department of Pharmaceutics, VT'sS. S. Jondhle College of Pharmacy, Asangoan, Tal. Shahapur, Dist. Thane-42160

Maharashtra, India. EMail:pharmajspatil@gmail. com

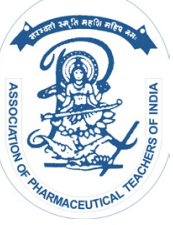

www.ijper.org 


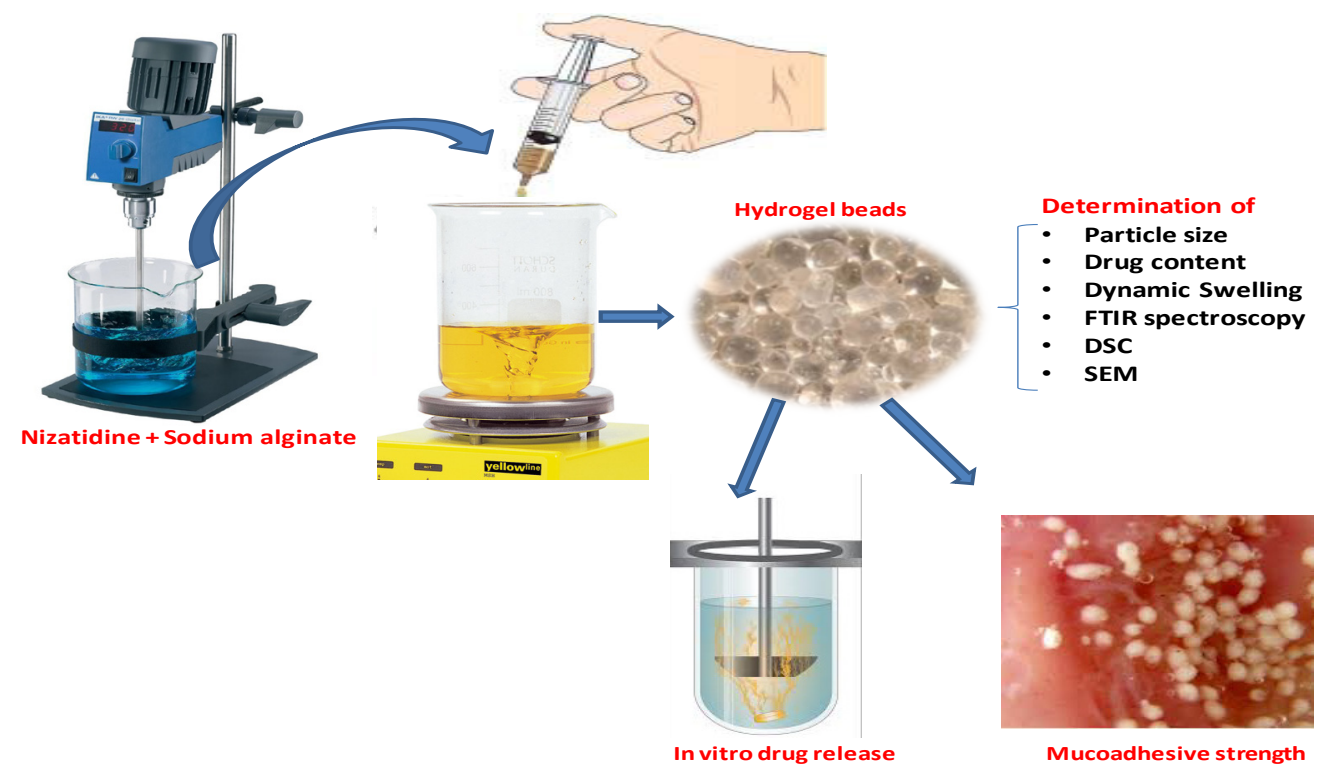

\section{Graphical Abstract}

symptoms. Five $\mathrm{H}_{2}$ receptor antagonists have been used worldwide for more than two decades includes cimetidine, ranitidine, famotidine, nizatidine and roxatidine. These drugs differ slightly in structure but have many similarities in their pharmacological properties. These agents only partially inhibit the acid secretion stimulated by gastrin and are more effective for inhibiting intra gastric acidity during periods of basal acid secretion.

Nizatidine is a competitive inhibitor of $\mathrm{H}_{2}$ receptor for gastric acid secretion and is used for the treatment of acid-reflux disorders, peptic ulcer, active benign gastric ulcer and active duodenal ulcers. It is having an oral bioavailability of $70 \%$ with a very short biological half life of 1-2 h. Moreover it is reported that nizatidine stimulates gastrointestinal motility. Hence, to increase the duration of GIT retention and drug release, sustained mucoadhesive hydrogel beads are an appropriate dosage form for drugs like nizatidine. Nizatidinedoes not have any demonstrable anti-androgenic effects and drug interactions compared to any other class of $\mathrm{H}_{2}$ receptor antagonists. It also finds applications in the field of local delivery of drug to the stomach and proximal small intestine and importantly in treating microorganisms $(H$. pylori) which colonize the stomach because the major factors governing reduced luminal drug delivery are gastric acidity, gastric emptying and the epithelial mucus layer and therefore it helps to provide better availability of new products with new therapeutic possibilities and increased patient compliance, ${ }^{2,3}$ in the present investigation we selected nizatidine as a model drug to formulate mucoadhesive multiparticulate hydrogel beads. This work focused on the preparation of novel nizatidine chitosan-alginate mucoadhesive beads with inner calcium

\section{Table 1: Formulation details, bead size and percent drug content of hydrogel beads}

\begin{tabular}{|c|c|c|c|c|c|c|}
\hline $\begin{array}{c}\text { Batch } \\
\text { Code }\end{array}$ & $\begin{array}{c}\text { Drug } \\
\text { (parts) }\end{array}$ & $\begin{array}{c}\text { Sodium } \\
\text { Alginate } \\
\text { (parts) }\end{array}$ & $\begin{array}{c}\text { Chitosan } \\
\text { (parts) }\end{array}$ & $\begin{array}{c}\text { Calcium } \\
\text { Chloride } \\
(\% \mathbf{w} / \mathbf{v})\end{array}$ & $\begin{array}{c}\text { Average } \\
\mathbf{s i z e}(\boldsymbol{\mu m})\end{array}$ & $\begin{array}{c}\text { Drug content } \\
(\%)\end{array}$ \\
\hline F1 & 1 & 1 & 1 & 1 & $728 \pm 2.12$ & $72.11 \pm 0.14$ \\
\hline F2 & 1 & 2 & 1 & 1 & $781 \pm 3.23$ & $76.38 \pm 0.37$ \\
\hline F3 & 2 & 1 & 1 & 1 & $801 \pm 1.99$ & $73.48 \pm 0.31$ \\
\hline F4 & 1 & 1 & 1 & 2 & $640 \pm 2.74$ & $74.33 \pm 0.16$ \\
\hline F5 & 1 & 2 & 1 & 2 & $699 \pm 3.64$ & $78.93 \pm 0.54$ \\
\hline F6 & 2 & 1 & 1 & 2 & $623 \pm 3.21$ & $74.85 \pm 0.36$ \\
\hline F7 & 1 & 1 & 1 & 3 & $612 \pm 3.51$ & $91.61 \pm 0.35$ \\
\hline F8 & 1 & 2 & 1 & 3 & $681 \pm 2.62$ & $76.53 \pm 0.19$ \\
\hline F9 & 2 & 1 & 1 & 3 & $624 \pm 3.72$ & $73.75 \pm 0.34$ \\
\hline F10 & 1 & 1 & -- & 2 & $695 \pm 2.91$ & $68.13 \pm 0.21$ \\
\hline F11 & 1 & 2 & -- & 2 & $723 \pm 2.56$ & $69.33 \pm 0.08$ \\
\hline F12 & 2 & 1 & -- & 2 & $699 \pm 3.12$ & $69.93 \pm 0.17$ \\
\hline
\end{tabular}


chloride cross-linked alginate core with outer chitosanalginate complex membrane and loaded in capsules. The one-stage procedure for the preparation of cross-linking reinforced chitosan-alginate beads was examined by dropping alginate solution into chitosan solution containing calcium chloride cross-linking agent.

\section{MATERIALS AND METHODS}

\section{Materials}

Nizatidineand chitosan were obtained asa gift sample from Dr. Reddy's Laboratory(Hyderabad, India) and Central Institute of Fisheries and Technology (Cochin, India), respectively. Sodium alginate was purchased from Loba Chemicals (Mumbai India). All other chemicals, reagents and solvents used were of pharmaceutical or analytical grade.

\section{Methods}

\section{Preparation of hydrogel beads ${ }^{4-6}$}

Ionotropic gelation technique has been widely used for microbeads preparation purpose. The natural polyelectrolytes are having a property of coating on the drug core and act as release rate retardants contains certain anions on their chemical structure. These anions forms meshwork structure by combining with the polyvalent cat ions and induce gelation by binding mainly to the anion blocks.

Hydrogel beads of nizatidine were prepared as per our previously reported article on hydrogel beads. ${ }^{6}$ Briefly, weighed quantity of nizatidine was dissolved in $15 \mathrm{ml}$ of deionized water in a beaker.

In another beaker sodium alginate was soaked for $3 \mathrm{~h}$, in measured amount of distilled water. Preparednizatidine solution was slowly added to the beaker containing sodium alginate with continuous stirring. The stirring was continued to obtain uniform dispersion of nizatidine in sodium alginate. The resultant homogeneous bubble free slurry dispersion was dropped through a $21 \mathrm{G}$ syringe needle into $100 \mathrm{ml}$ of calcium chloride solution with or without containing chitosan (Table 1), which was kept under stirring to improve the mechanical strength of the beads and to prevent aggregation of them. After $15 \mathrm{~min}$, the formed beads were collected by filtration and dried at $40^{\circ} \mathrm{C}$ overnight. The dried beads were doubly wrapped in an aluminum foil and kept in a desiccator till further use.

\section{Evaluation Parameters of Microbeads}

Particle size measurement ${ }^{7,8}$

All the particulate formulations were subjected for particle size analysis using a digimatic micrometer (MDC-
25S Mitutoyo, Tokyo, Japan) having an accuracy of $0.001 \mathrm{~mm}$. The average diameter of randomly selected 100 particles from all the formulations was measured.

\section{Drug content estimation ${ }^{9}$}

Known amount of beads $(10 \mathrm{mg})$ were added to $10 \mathrm{ml}$ phosphate buffer (USP) of $\mathrm{pH} 7.4$ and $\mathrm{pH} 1.2$ solutions separately for complete swelling at $37^{\circ} \mathrm{C}$. The beads were crushed in a glass mortar with pestle the solution was than kept for $2 \mathrm{~h}$ to extract the drug completely and centrifuged to remove polymeric debris. The clear supernatant solution was analyzed for drug content at $317.6 \mathrm{~nm}$ using UV-visible spectrophotometer (Pharmaspec-UV/ Visible spectrophotometer-1700, Simadzu, Japan). The amount of nizatidine present in microbeads was determined using calibration curve and the following formula:

Percent drug content $=$ Practical concentration $/$ Theoretical concentration $\times 100$

\section{Dynamic swelling study ${ }^{10,11}$}

Hydrogels exhibit dynamic swelling property. This unique nature enables to release the entrapped drug from hydrogels, because swelling is directly proportional to drug release. The pores of matrix network opens due to swelling of hydrogel beads and release of the entrapped solute occurs. Therefore, the dynamic swelling study of the prepared beads was carried out by mass measurement as a function of $\mathrm{pH}$. The degree of swelling was measured gravimetrically by weighing the particles prior and after swelling. Weighed quantity of the dried microbeads dose equivalent to marketed nizatidine formulations were immersed for $2 \mathrm{~h}$, in $\mathrm{pH} 1.2$ buffer as swelling medium. Then swelling medium was replaced with $\mathrm{pH} 7$. 4 phosphate buffer and kept until to reach equilibrium. Subsequently, microbeads were removed from the buffer solution, carefully blotted with tissue paper and weighed. The degree of swelling (swelling index) was calculated using theformua: $\frac{W_{1}}{W_{1}} \square 100$

Where, $W_{1}$ is mass of the dry beads and $W_{2}$ is the mass of swollen beads. Each swelling experiment was repeated three times, and the average value was taken as the percentage swelling value.

\section{Mucoadhesive strength determination ${ }^{12-14}$}

The mucoadhesive property of microbeads was evaluated by an in vitro adhesion testing method known as the wash-off test. Freshly excised pieces of sheep intestinal mucosa obtained from local slaughter house were 


\section{Percentage swelling Index}

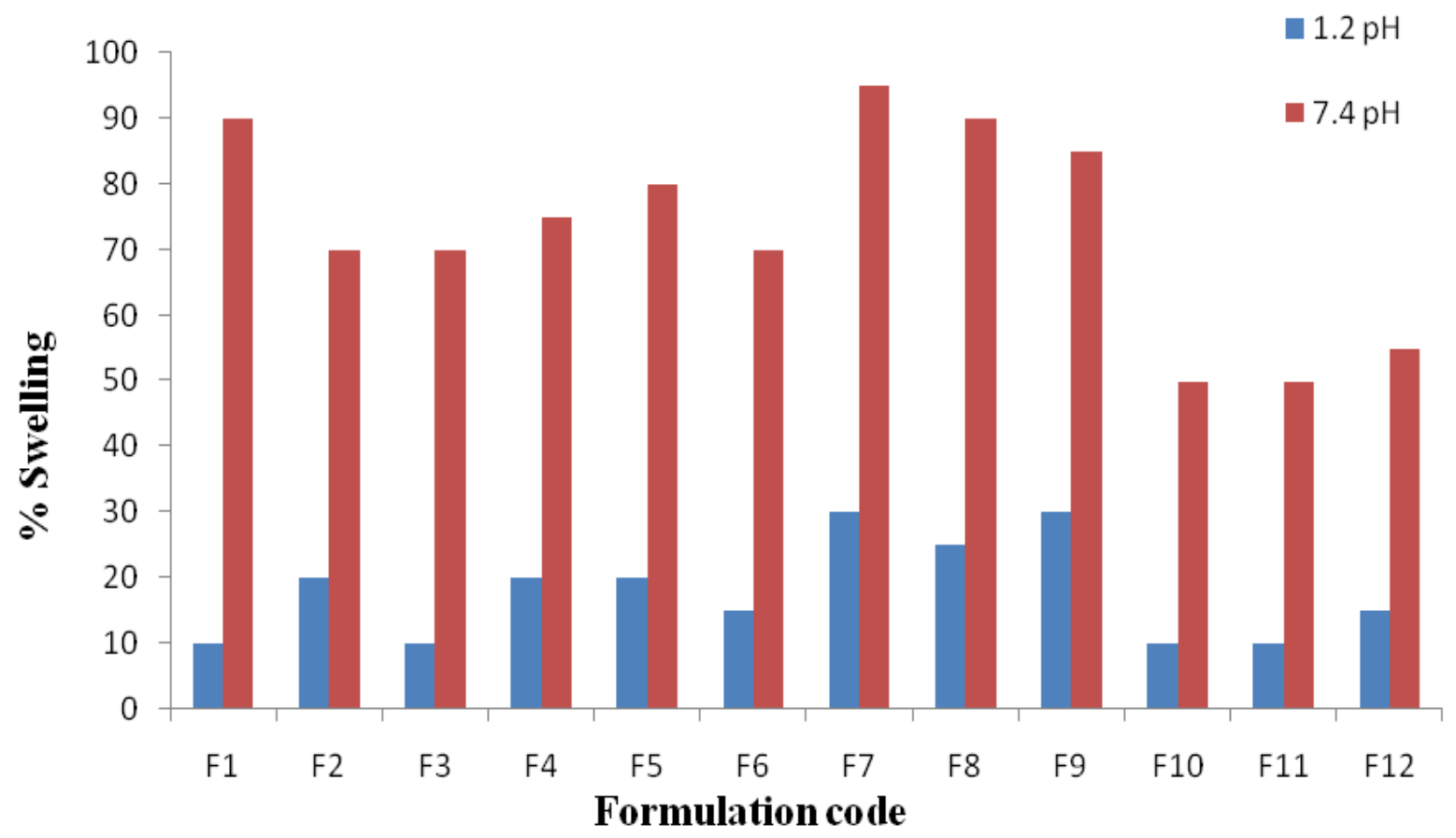

Figure 1: Bar graph profile showing percentage swelling index of hydrogel beads formulations percentage swelling

mounted onto glass slides. About 100 microbeads were spread onto wet rinsed tissue specimen and immediately thereafter the slides were suspended onto the arm of a tablet disintegrating machine containing $7.4 \mathrm{pH}$ phosphate buffer solutions at $37^{\circ} \mathrm{C}$. The tissue specimen was given a slow, regular up and down movement in the test fluid for $8 \mathrm{~h}$. At the end of 1, 2, 3, 4, 5, 6, 7, $8 \mathrm{~h}$ the machine was stopped and the number of microbeads still adhering to the tissue were counted. The percent mucoadhesive strength was calculated using the equation:

$$
\% \text { mucoadhesive strength }=(\mathrm{Na}-\mathrm{Nl}) / \mathrm{Na} \times 100
$$

Where, $\mathrm{Na}=$ number of microspheres applied; $\mathrm{Nl}=$ number of microspheres leached out

\section{In vitro drug release study ${ }^{15-17}$}

In vitro drug release studies were performed on the beads, using a dissolution apparatus (USP-XXIII, Electro lab, Mumbai). A weighed amount of individual bead formulations were added to muslin cloth, placed in a basket and dipped indissolution vessel containing 900 $\mathrm{ml}$ of $\mathrm{pH} 1.2$ phosphate buffer for $2 \mathrm{~h}$ and fallowed by $\mathrm{pH} 7.4$ phosphate buffer till end of the study at 37.0 $\pm 0.5^{\circ} \mathrm{C}$ with $50 \mathrm{rpm}$ paddle speed. At set times, $5 \mathrm{ml}$ aliquots were withdrawn, filtered and amount of drug released was assayed spectrophotometrically at 317.6 $\mathrm{nm}$. The same amount of medium was replaced with fresh buffer solution. The release data were fitted to various mathematical models to know which model is best fitting the obtained release profile.

\section{FTIR Spectroscopy ${ }^{18}$}

The spectra of nizatidine and its different formulations were recorded on FTIR spectrometer (Bruker-Alpha) and evaluated for compatibility within the formulations.

\section{Differential Scanning Calorimetry (DSC) ${ }^{18}$}

DSC allows the fast evaluation of drug-polymer compatibility because it shows changes in the appearance, shift of melting endotherms and exotherms and/or variation in the corresponding enthalpies of reaction. Thermal behavior of the beads was examined by using a thermal analyzer (DSC-60 Shimadzu, Japan). The DSC thermograms of pure nizatidine and the formulations were recorded on a thermal analyzer. The thermal analysis was performed at a heating rate of $10^{\circ} \mathrm{C} / \mathrm{min}$ over temperature range of $50-400^{\circ} \mathrm{C}$ under a nitrogen atmosphere in a micro calorimeter and then thermograms were obtained.

\section{Scanning Electron Microscopy (SEM) ${ }^{5}$}

For visualizing surface morphology of beads SEM is promising technique. Also by taking microphotographs at time intervals from dissolution study we can predict about possible release pattern of particulate drug delivery system. The surface morphology of the beads was investigated using scanning electron microscope (JEOL, 


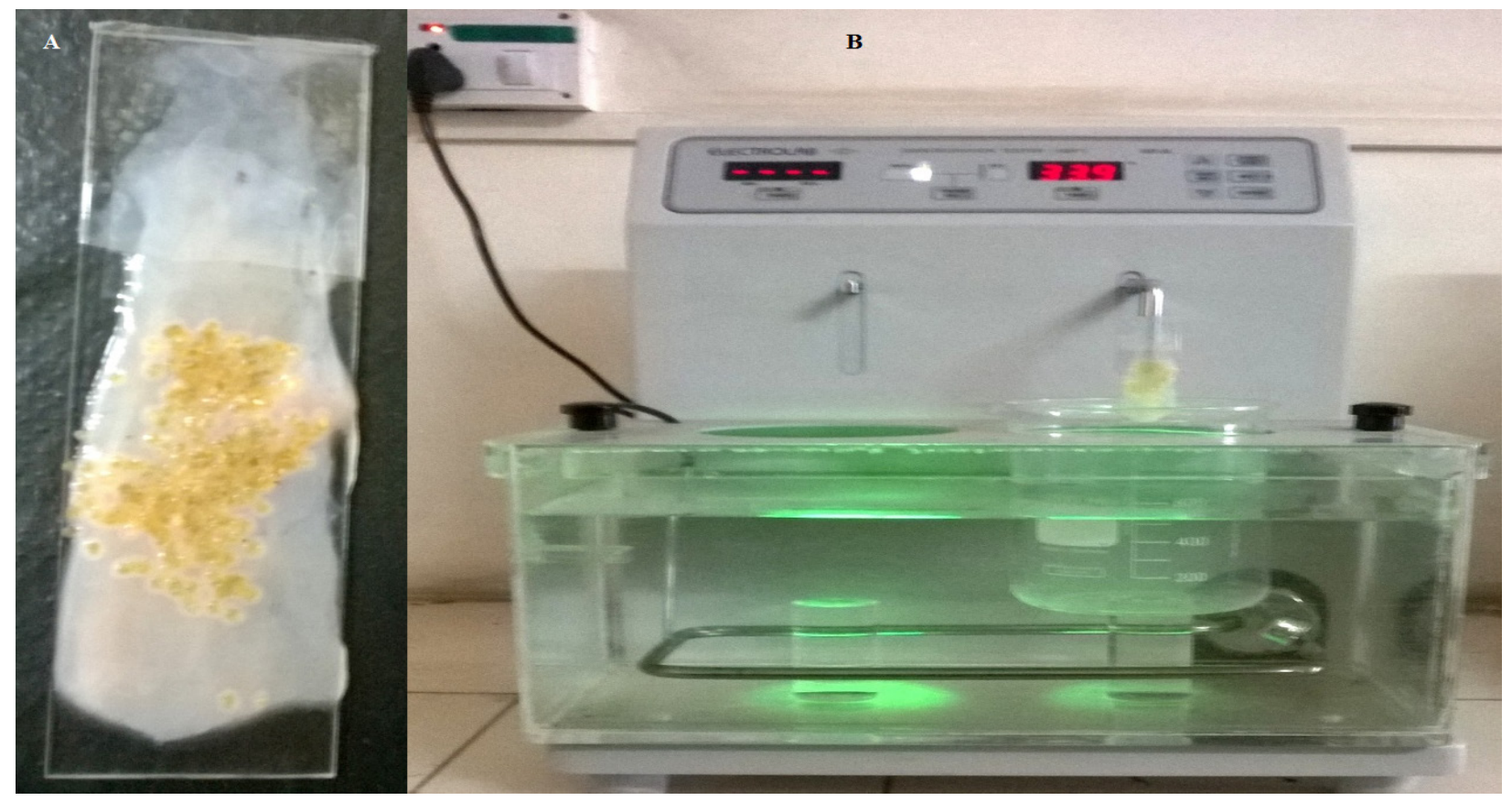

Figure 2: In vitro Wash off test for mucoadhesive hydrogel beads Apparatus used for the study

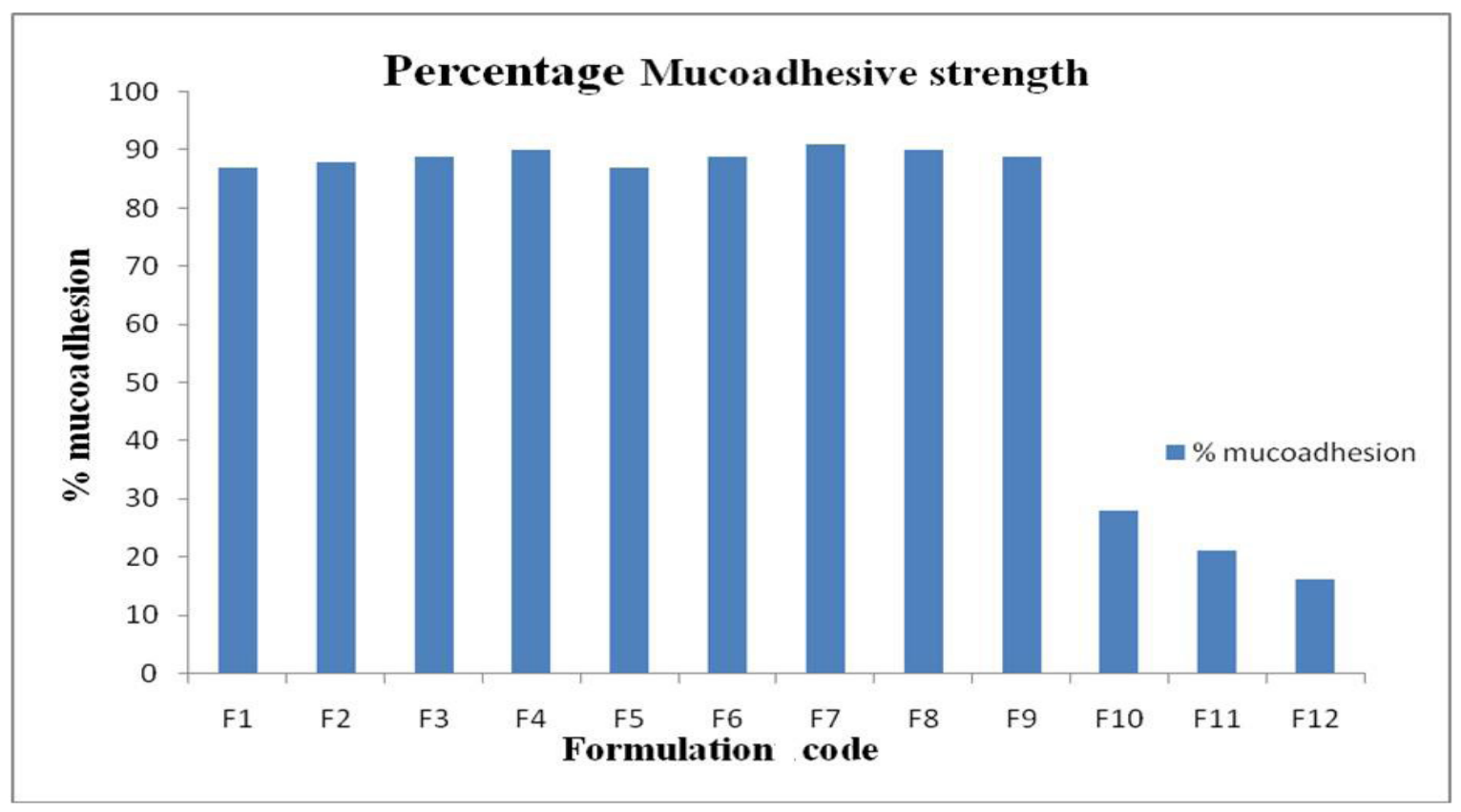

Figure 3: Strength of hydrogel beads formulations F1 to F12

JSM-35CF, Japan). The beads were mounted onto stubs using double sided adhesive tape and sputter coated with platinum using a sputter coater. The coated beads were observed under SEM instrument at the required magnification at room temperature. The acceleration voltage used was $10 \mathrm{kV}$ with the secondary electron image as a detector.

\section{RESULT AND DISCUSSION}

The details of formulation composition, results of bead size and percent drug content of various batches are shown in Table 1. The prepared hydrogel microbeads were smooth and free flowing with light brownish in colour. The percent drug content was found to be in the range of $68.13 \pm 0.21$ to $91.61 \pm 0.35 \%$. The formulations coated with chitosan showed higher drug con- 
A

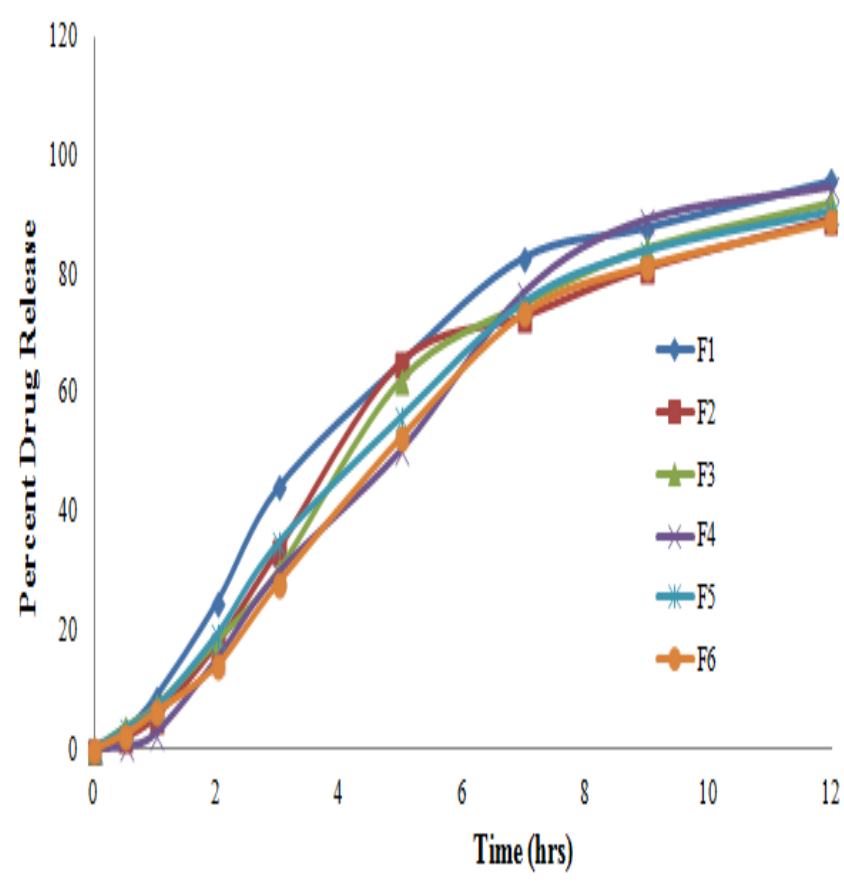

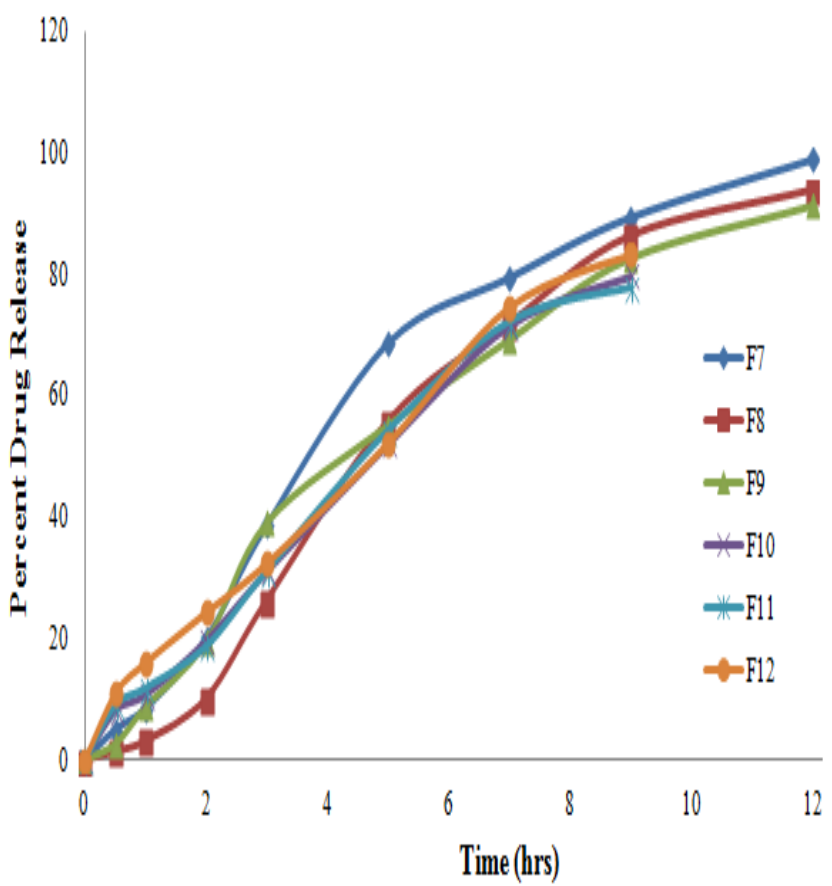

Figure 4: In vitro drug release profile of hydrogel formulations

A: Batches F1 to F6, B: Batches F7 to F12

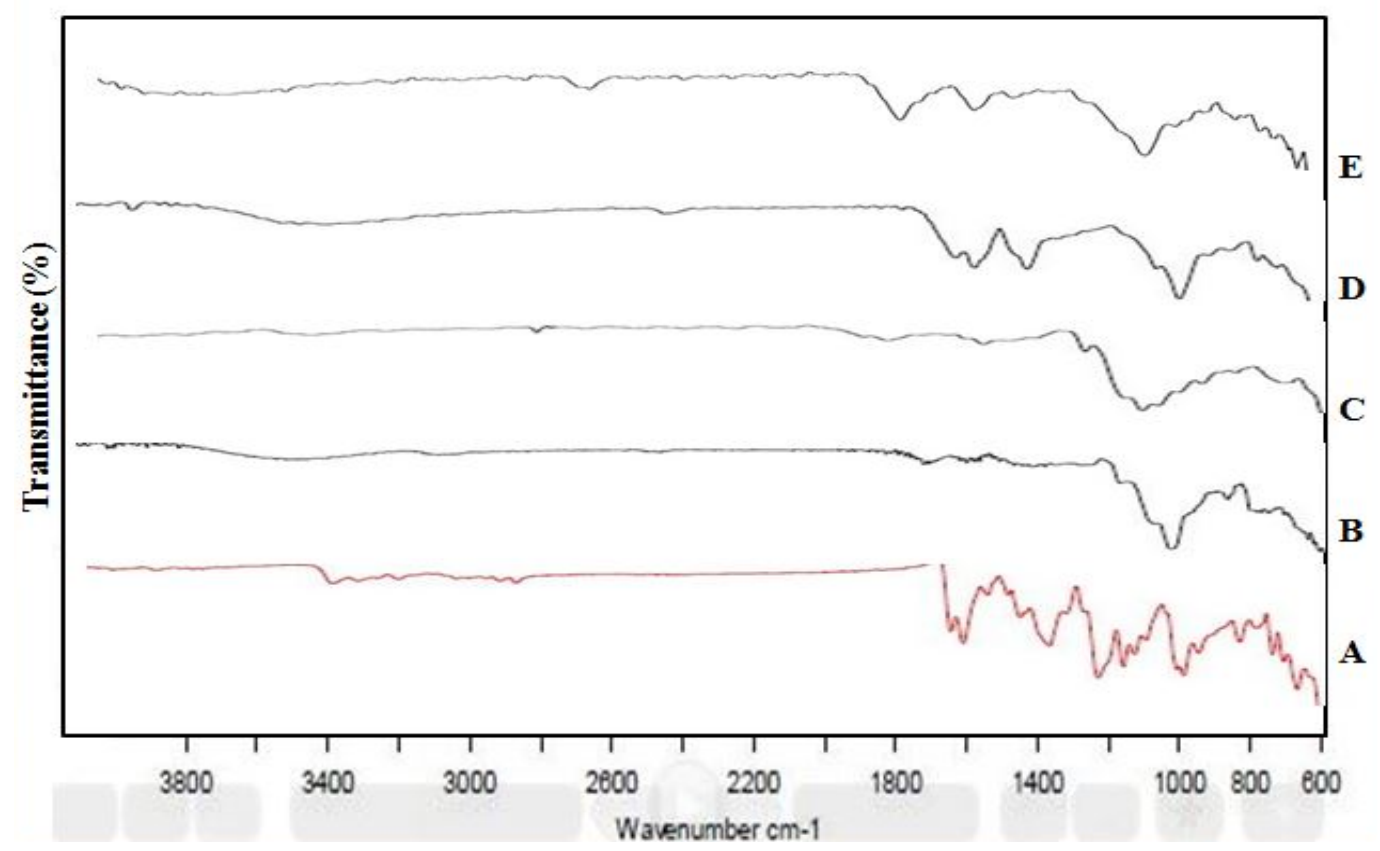

Figure 5: FTIR Spectra of pure drug and formulations

A:Nizatidine, B: Sodium alginate, C: Chitosan, D: Drug loaded beads with alginate and chitosan, E: Drug loaded beads with sodium alginate

\section{Table 2: Kinetic values of nizatidine realease from optimized microbead formulation.}

\begin{tabular}{|c|c|c|c|}
\hline Formulations code & Zero order Equation & Korsemayer Peppas Equation & Higuchi Equation \\
\hline \multirow{2}{*}{ F5 } & $\mathrm{y}=8.3913 \mathrm{x}+4.2127$ & $\mathrm{y}=-0.12 \mathrm{x}+1.9537$ & $\mathrm{y}=30.926 \mathrm{x}-14.624$ \\
& $\mathrm{R}^{2}=0.9448$ & $\mathrm{R}^{2}=0.8915$ & $\mathrm{R}^{2}=0.9489$ \\
\hline \multirow{2}{*}{ F7 } & $\mathrm{y}=9.0354 \mathrm{x}+5.7378$ & $\mathrm{y}=0.0628 \mathrm{x}+2.1273$ & $\mathrm{y}=33.478 \mathrm{x}-14.864$ \\
& $\mathrm{R}^{2}=0.9838$ & $\mathrm{R}^{2}=0.9812$ & $\mathrm{R}^{2}=0.9477$ \\
\hline
\end{tabular}




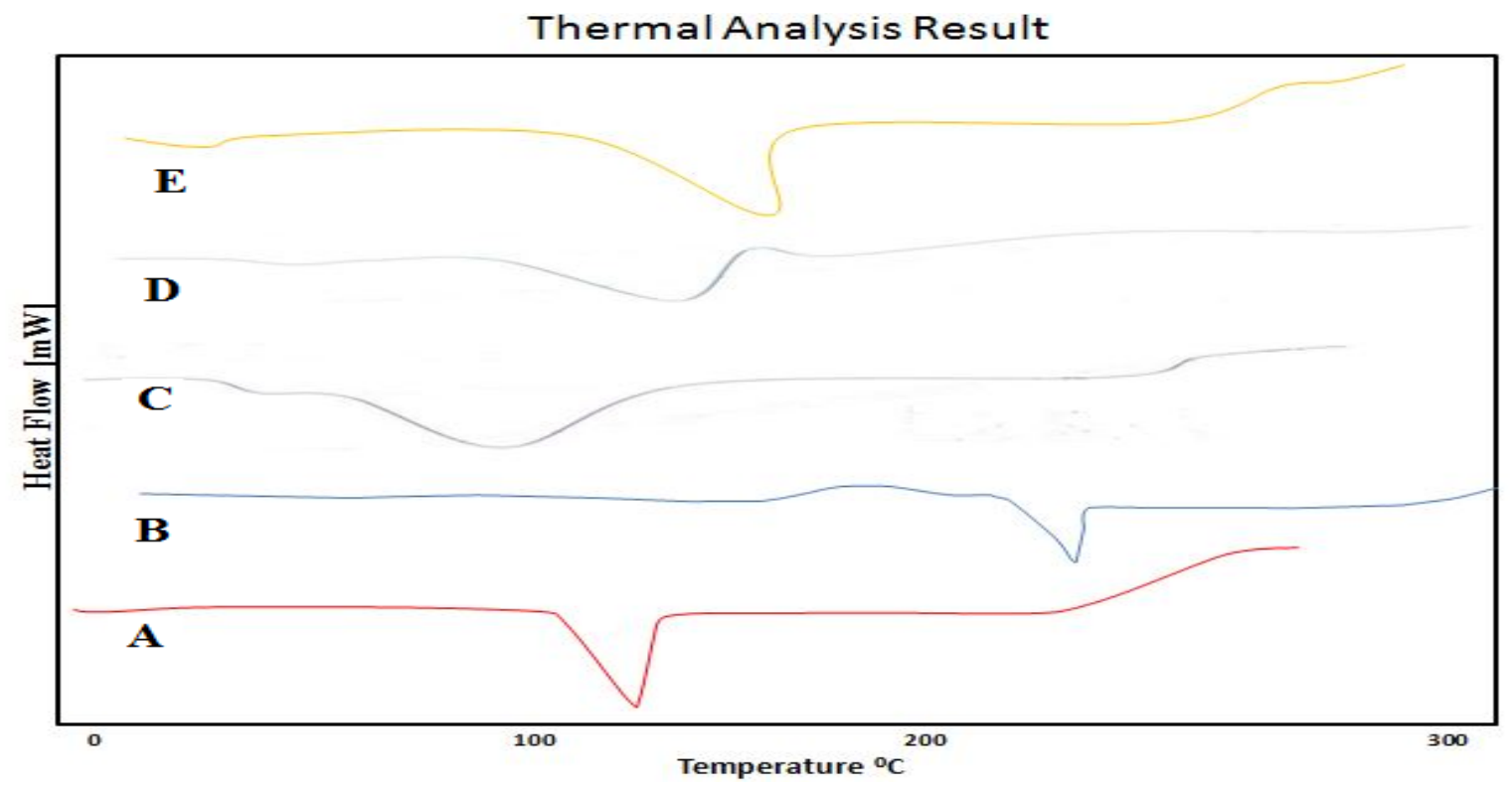

Figure 6: DSC thermograms of pure drug, polymers and formulations

A:Nizatidine, B: Sodium alginate, C: Chitosan, D: Drug loaded beads with alginate and chitosan, E: Drug loaded beads with sodium alginate
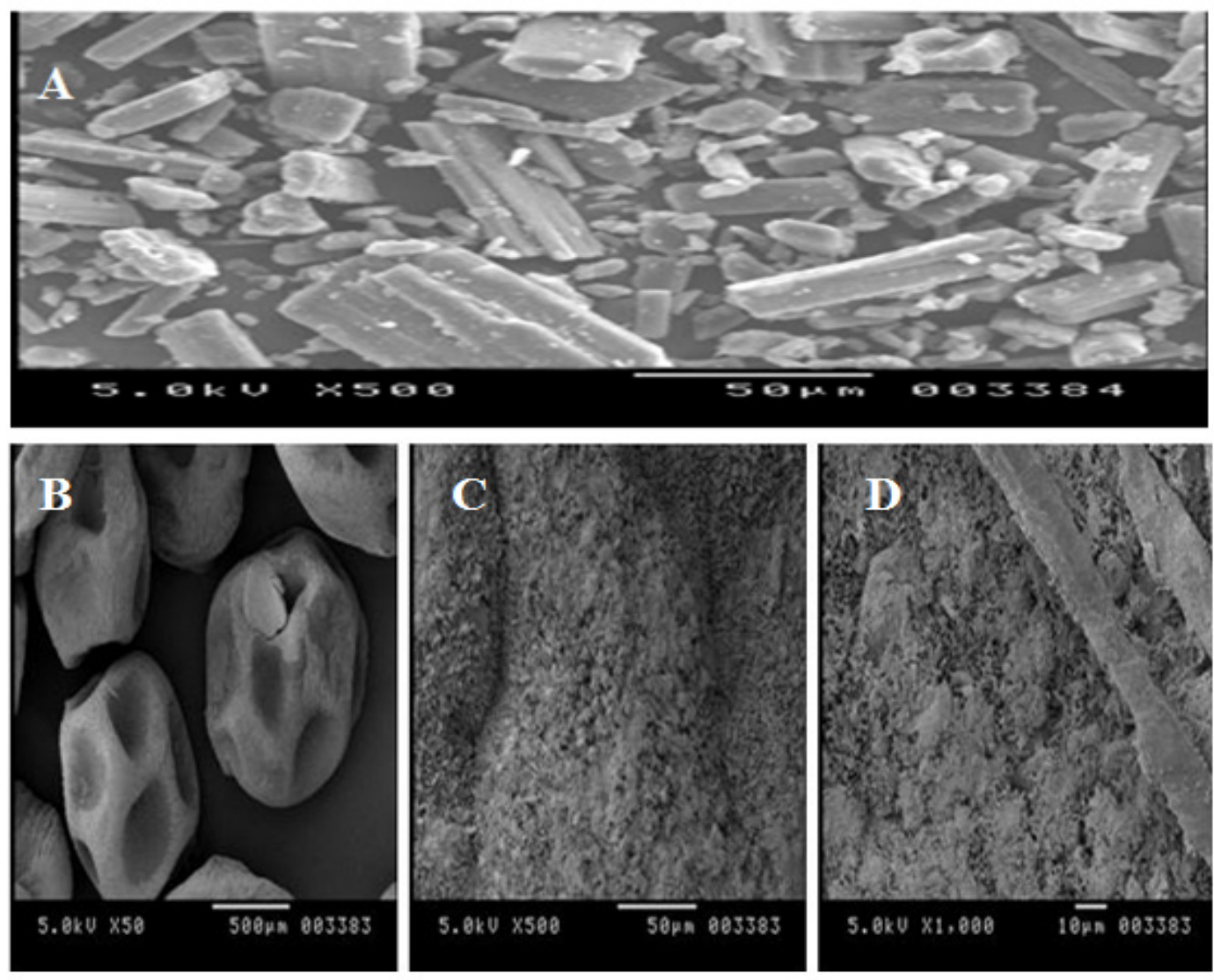

Figure 7: SEM Microphotographs batch containing Drug loaded alginate-chitosan beads A:Pure drug, B:Microbeads in bulk, C:Surface morphology at 500X, D: Surface morphology at 1000X

tent when compared to the formulations which are not coated with chitosan. We analyzed the particle size of prepared hydrogel beads, which was found in the range of $612 \pm 3.51$ to $801 \pm 1.99 \mu \mathrm{m}$ for different batches.
The results indicated that the size of hydrogel beads found to be increased proportionally as the amount of alginate concentration increased. This could be attributed to the increase in micro-viscosity of the polymeric 

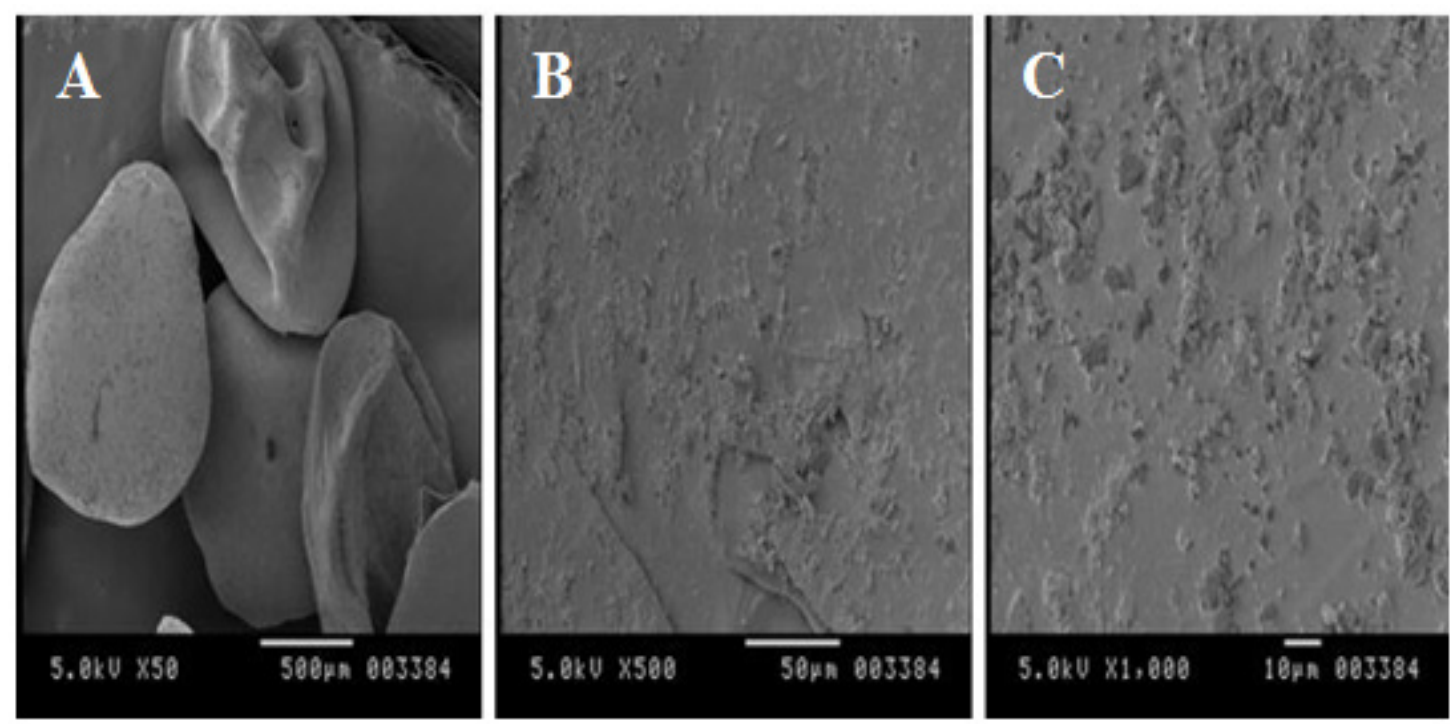

Figure 8: SEM Microphotographs of batch containing drug loaded alginate beads without chitosan A: Microbeads in bulk, B: Surface morphology at 500X, C: Surface morphology at 1000X

dispersion due to increased alginate concentration, which eventually led to formation of bigger beads.

The swelling ability of alginate-chitosan complex hydrogel beads is dependent on $\mathrm{pH}$ value of the swelling medium. Formulations were allowed to swell for initial $2 \mathrm{~h}$ in acid buffer $\mathrm{pH} 1.2$ solution, followed by in phosphate buffer $\mathrm{pH} 7.4$ solution up to $12 \mathrm{~h}$. The details of swelling profile are presented in Figure 1. Formulation F7 with alginate-chitosan complex showed highest swelling index of 95\%, while formulations F10 and F11 without chitosan showed lowest swelling index of $50 \%$. Cross-linked hydrogel beads showed obviously the higher swelling ability at $\mathrm{pH} 1.2$ and slightly higher swelling ability at $\mathrm{pH}$ 7.4. The protonation of outer chitosan coat in presence of acidic medium could be attributed to the increased swelling ability of formulations and on other hand the slightly increased swelling degree of hydrogel beads at alkaline $\mathrm{pH}$ is attributed to the ionization of carboxyl groups of alginate in the inner complex layer of the beads. Swelling ability of hydrogel beads not only depends on $\mathrm{pH}$ of medium used but also on the concentration of matrix forming polymers and the strength of cross linking agent used.

Mucoadhesive strength study or In vitro wash off test was carried out by using sheep intestinal mucosa. The details of procedure followed and data obtained are presented in Figure 2 and 3 respectively. Alginate-chitosan complex hydrogel beads exhibited good mucoadhesive strength, which might be due to ionization of chitosan outer coat, increased the mucoadhesion ability of beads, whereas, the formulations without chitosan showed very poor mucoadhesion ability due to absence of chitosan coat on their outer surface. The formulation F7 showed highest mucoadhesive strength of $91 \%$, while F12 batch showed lowest mucoadhesive strength of $16 \%$. The in vitro wash off results revealed that the hydrogel beads are able to adhere to the mucous membrane for longer time and release drug from the microbeads slowly for an extended period of time up to $12 \mathrm{~h}$.

The in vitro drug release profiles of all the batches are shown in Figure 4. The formulation F1 and F7 showed highest drug release of $95.79 \%$ and $98.87 \%$ respectively. All the alginate-chitosan complex formulations showed slower drug release might be due to rate retarding property of outer chitosan coat and slower erosion diffusion of drug from the beads. The results of drug release profile revealed that the rate and extent of drug release from-Preparation of hydrogel beadssignificantly decreased with an increase in polymer concentration. This could be attributed to the increase of alginate matrix density and in the diffusion path length which the drug molecules have to traverse. The burst effect in drug release was characterized by an initial phase of high release from these beads. However, as gelation proceeded through cross-linking of alginate with calcium ions, the remaining drug was released at a slower rate followed by a phase of moderate release. This biphasic pattern of release is a characteristic feature of matrix diffusion kinetics. ${ }^{19}$ The initial burst effect was considerably reduced with the increase in alginate gum concentration. The initial burst effect from batches of chitosan-coated beads was considerably reduced when compared to the corresponding batches of non-coated beads. The fact is that chitosan coating over the beads resulted in better incorporation efficiency and formed a 
thick coating layer around the beads. This could be the reason for the observed decrease in the burst effect. The drug release found to be slow and extended up to $12 \mathrm{~h}$ as increase in the concentration of cross-linking agent calcium chloride. Low concentration of calcium chloride leads probably to a loose gel. As a consequence, the drug can be easily released from the beads, as the steric entanglements do not constitute a strong barrier. Further increase in the concentration of calcium chloride gives more structured gel and the drug is more retained inside the beads due to steric reason, since the existence of physical entanglements of cross-linked alginate-calcium chloride complex of lower dimensions controlling the drug diffusion flow within the beads. ${ }^{20}$ At high concentration of calcium chloride, as in formulation F7, strong and rigid gel is formed around the matrix and this strong gel does not allow the dissolution medium to penetrate into the matrix at a high speed, resulting in a reduction in the release rate.

In order to describe the kinetics of drug release from SR preparations, various mathematical equations have been proposed. The zero order models describe the system, where the drug release is independent of its concentration. According to Higuchi model, the drug release from matrix is directly proportional to square root of time and is based on the Fickian diffusion. A more comprehensive, but still very simple, semi-empirical equation to describe drug release mechanism from polymeric systems more precisely is the so-called Korsmeyer-Peppaspower law. To study the mechanism of drug release from the randomly selected formulation F5 and F7 the release data was fitted to above mentioned models. ${ }^{20}$ The details of data obtained are presented in Table 2 . The formulation F7 showed quasi Fickian release pattern with $\mathrm{n}=0.0628$ and regression of $\mathrm{R}^{2}=0.9812$. The Higuchi plot of F5 formulation showed good release profile with $\mathrm{R}^{2}=0.9489$. The embedded drug within the microbeads showed matrix and SR mechanism, from this matrix drug was diffused as indicated by Higuchi plot. ${ }^{21-24}$ In an effort to investigate the possible incompatibilities between drug and polymer, we have carried out FTIR and thermal analysis of pure nizatidine, polymers and drug-loaded beads. The FTIR spectra of formulation (Figure 5) revealed that the characteristic peaks of drug were retained in the formulation containing polymers indicated that the drug is intact in the formulation. The DSC thermogram (Figure 6) showed sharp endothermic peak at $135.91{ }^{\circ} \mathrm{C}$ which corresponds to the melting point of pure drug. The peak starts at $130.42^{\circ} \mathrm{C}$ and end at $145.9^{\circ} \mathrm{C}$ which indicates the higher melting rate of nizatidine. The sodium alginate and chitosan showed characteristic peak at 249.02 and $100.02^{\circ} \mathrm{C}$ respectively, which corresponds to their melting point. The absence of detectable peak of nizatidine in the formulation F7 and F12 clearly indicates that nizatidine was dispersed completely in the formulation, thus modifying the microbeads to an amorphous, disordered crystalline phase. The formulation F7 showed endothermic peak at $140.11^{\circ} \mathrm{C}$ which revels slight shifting of melting point of nizatidine within the formulation. So nizatidine found to be compatible with the polymers in this formulation.

The surface morphology was examined by SEM studies (Figure 7 and 8). SEM microphotograph of pure nizatidine showed crystalline nature. The SEM microphotographs revealed that the beads were irregular in shape having smooth and dense surface with inward dent and shrinkage due to the collapse of the wall of the beads during dehydration. The fibrous network was also found on the surface of the beads. The microbeads from formulation $\mathrm{F} 7$ contain nizatidine, sodium alginate and chitosan (Figure 7), showed debris or dimple like structure on their surface. This might be due to chitosan undergo less surface deformation due to sodium alginate shrinkage during drying. Exposure of sodium alginate to heat during drying evaporates excessive interstitial solvent that leaves debris. In Figure 7 (B), microbeads from same formulation showed a rough surface morphology that indicates formation of micropores due to solvent evaporation, which was also observed in Figure 7 (C). The microbeads from batch F12 contain nizatidine and sodium alginate without chitosan (Figure 8 (A), showed smooth surface and debris due to shrinkage of sodium alginate during drying. The Figure 8 (B), showed smooth surface and less micropores as compared to microbeads coated with chitosan. The absence of chitosan in the formulation showed smooth surface and fewer micropores on the surface.

\section{CONCLUSION}

From the experimental results it can be concluded that FTIR and thermal studies of formulations reveled that nizatidine was compatible with all the polymers used. From swelling index study it was proved that alginatechitosan complex formulation showed better swelling effect. From in vitro mucoadhesive strength it was evident that the alginate-chitosan complex formulation showed better mucoadhesive strength when compared to formulations without chitosan coating. In vitro drug release study of alginate-chitosan complex formulation indicated that nizatidine was released in sustained manner up to $12 \mathrm{~h}$ with best zero order profile and quasi Fickian release pattern. The embedded drug within the 
microbeads showed matrix and controlled release mechanism, from this matrix drug was diffused as indicated Higuchi plot. Hence, it can be concluded from the study that, among the prepared formulations with respect to percentage drug content, swelling studies and in vitro drug release, the alginate-chitosan beads prepared by ionotropic gelation and polyelectrolyte complexation method found to be better than ionically cross linked alginate beads alone. Therefore, dual cross-linked beads are promising carrier for oral control release.

\section{ACKNOWLEDGEMENT}

Authors are thankful to Mr. Shivsharan B. Dhadde, Assistant Professor, VT's Shivajirao S. Jondhle Col- lege of Pharmacy, Asangaon for helping in the preparation and proof reading of this manuscript.

\section{CONFLICTS OF INTEREST}

Authors disclose that there is no Conflict of Interest in this work.

\section{ABBREVIATION}

DSC : Differential scanning calorimetry

FTIR : Fourier transforms infrared spectroscopy

GIT :Gastro intestinal tract

SEM : Scanning electron microscopy

\section{SUMMARY}

- Mucoadhesive hydrogel beads of nizatidine were prepared with or without chitosan by using sodium alginate as polymer

- Formulations with chitosan showed good drug content, swelling index and mucoadhesive strength when compared to batches containing alginate alone.

- The drug in formulations found to be intact and compatible with polymers used which was confirmed by DSC and FTIR analysis. Surface morphology of prepared beads were found satisfactory which was confirmed by SEM

- Two optimized batches containing alginate-chitosan shows Higuchi model and perfect zero order release. All the batches with copolymer showed better sustained the drug release more than $12 \mathrm{~h}$ when compared with batches prepared with alginate alone.

\section{About Authors}

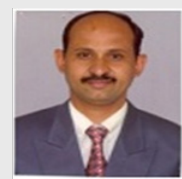

Dr. Jagadevappa S. Patil, is an Professor and Principal at the Department of Pharmaceutics, VT's, Shivajirao S. Jondhle College of Pharmacy, Asangaon. Dr. Patil received his PhD degree from Rajiv Gandhi University of Health Sciences, Karnataka, Bangalore. His research interest is in the area of Site-specific delivery of drugs for optimization of drug therapy, development of nanoparticles, microparticles and liposomes. He is serving as Editorial Board Member and reviewer for many reputed journals and he has more than 50 publications in national and international journals. Dr Patil, received several awards, scholarship and research grant from the different agencies.

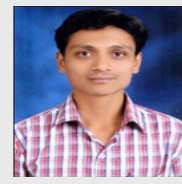

Mr. Kailash V. Vilegave was born on 17 April 1985 in, Latur, Maharashtra, India. He completed his B. Pharm. in 2007 \& M. Pharm. (Pharmaceutics) in 2010 from Rajiv Gandhi University of health science, Karnataka, Bangalore, India. He is serving as an Assistant Professor in the department of Pharmaceutics at Shivajirao S. Jondhle College of Pharmacy, Mumbai, Maharashtra, India, since July 2010. He has more than 15 publications in national and international journals. His research interest is in the area of novel drug delivery systems such as nanoparticles, microparticles and liposomes and their design, preparation optimization and evaluation.

\section{REFERENCES}

1. Abdul A, Varun J. Formulation, development and in vitro evaluation of candesartan cilexetil mucoadhesive microbeads. Int J Curr Pharm Res. 2012; 4(3): 109-18.

2. Jia-Qing HMD, Richard HH. Pharmacological and pharmacodynamic essentials of $\mathrm{H}_{2}$-receptor antagonists and proton pump inhibitors for the practicing physician. Best practice Res clingastroenterol 2001; 15(3): 355-70.
3. Takeuchi K, Kawauchi S, Araki H, Ueki S. Stimulation by nizatidine, a histamine $\mathrm{H}_{2}$ - receptor antagonist, of duodenal $\mathrm{HCO}_{3}$ - secretion in rats: relation to anticholinesterase activity. World J. Gastroenterol. 2000; 6(5): 651-8.

4. Patil JS, Kamalapur MV, Marapur SC, Kadam DV. Ionotropic gelation and polyelectrolyte complexation: The novel techniques to design hydrogel particulate sustained, modulated drug delivery system: A review. Dig J Nanomat Biostruct. 2010; 5(1): 241-8.

5. Patil JS, Kamalapur MV, Marapur SC, Shiralshetti SS, Kadam DV. lonotropically gelled chitosan-alginate complex hydrogel beads: Preparation, 
characterization and in vitro evaluation. Indian J Pharm Edu Res. 2012; 46(1): 248-52.

6. Patil JS, Kamalapur MV, Marapur SC, Shiralshetti SS. Ionotropically gelled novel hydrogel beads: Preparation, characterization and in vitro evaluation. Indian J. Pharm. Sci. 2011; 73(5): 504-09.

7. Hemalatha K, Lathaeswari R, Suganeswari M, Senthil KV, Anto SM. Formulation and evaluation of metoclopramide hydrochloride microbeads by ionotropic gelation method. Int J Pharm Bio Arch. 2011; 2(3): 921-5.

8. Grant GT, Morris ER, Rees DA, Smith PJC, Thom D. Biological interactions between Polysaccharides and Divalent cationsthe:egg-box model. FEBS Letters 1973; 32(1): 195.

9. Smrdel P, et al. The influence of selected parameters on the Size and Shape of Alginate beads prepared by ionotropic gelation. Sci Pharm. 2008; 76(1): 77-89.

10. Nayak AM, Hasnain MS, Beg S, Alam MI. Mucoadhesive beads of gliclazide: design, development and evaluation. Sci Asia. 2010; 36(1): 319-25.

11. Somashree R, et al. Preliminary investigation on the development of diltiazem resin complex loaded carboxymethyl xanthane beads. AAPS Pharm Sci Tech. 2008; 9(1): 295-301.

12. Muzaffar F, Murthy NV, Paul P, Semwal R. Formulation and evaluation of mucoadhesive microspheres of Amoxicillin trihydrate by using eudragit RS 100. Int J Chem Tech Res. 2010; 2(1): 466-70.

13. Hardenia SS, Jain A, Patel R, Kaushal A. Formulation and evaluation of mucoadhesive microspheres of Ciprofloxacin. J Adv Pharm Edu Res. 2011; 1(4): 214-24.

14. Bhanja S, Sudhakar M, Neelima V, Roy H. Development and evaluation of mucoadhesive microspheres of Irbesartan. Int J Pharm Res Health Sci. 2013; 1: 17-26.
15. Brahmaiah B, Sasikanth $\mathrm{K}$, Sreekanth N, Krishna C. Formulation and Evaluation of Extended Release Mucoadhesive Microspheres of Rosuvastatin. Int J Bio Pharm Res. 2013; 4(4): 271-81.

16. Thulasi VM, Sajeeth Cl. Formulation and Evaluation of Sustained Release Sodium alginate Microbeads of Carvedilol. Int J Pharm Tech Res. 2013; 5(4): 746-53.

17. Ghareeb MM, Issa AA, Hussein AA. Preparation and characterization of Cinnarizine floating oil entrapped calcium alginate beads. Int J Pharm Sci Res. 2012; 3: 501-8.

18. Klaus Florey. Analytical profiles of drug substances. Volume $19,1^{\text {st }}$ edition Elsevier publications; 397-427.

19. Rajinikanth PS, Sankar C, Mishra B. Sodium alginate microspheres of metoprololtartarate for intranasal systemic delivery: development and evaluation. Drug Deliv. 2003; 10(1): 21-8.

20. Verma A, Sharma M, Verma N, Pandit JK. Floating alginate beads: studies on formulation factors for improved drug entrapment efficiency and in vitro release. Farmacia 2013; 61(1): 143-61.

21. Higuchi WI. Analysis of data on the medicament release from ointments. $J$ Pharm Sci. 1962; 51(8): 802-4.

22. Costa P, Lobo JS. Modeling and comparison of dissolution profiles. Eur J Pharm Sci. 2001; 13(2): 123-33.

23. Sherina VM, Santhi K, Sajeeth Cl. Formulation and evaluation of sodium alginate microbeads as a carrier for the controlled release nifedipine. Int $\mathrm{J}$ Pharm Chem Sci. 2012; 1: 699-710.

24. Farhana Y, Talukdar MU, Islam MS, Laila S. Evaluation of aceclofenac loaded agarose beads prepared by ionotropic gelation method. Stamford J Pharm Sci. 2008; 1(1): 10-7. 